\title{
PLAN ESTRATÉGICO DE TECNOLOGÍAS DE LA INFORMACIÓN ORIENTADO AL ÉXITO DE PROYECTOS EN INSTITUCIONES DE EDUCACIÓN SUPERIOR
}

\author{
Vanessa Maribel Choque Soto ${ }^{1, \mathrm{a}, \mathrm{e}}$ \\ ${ }^{1}$ Departamento de Ingeniería de Sistemas, Universidad Andina del Cusco, Cusco, Perú. \\ ${ }^{a}$ Maestra en Gestión e Innovación Tecnológica \\ ”vchoque@uandina.edu.pe
}

Information technology strategic planning for the success of institutions of higher education

\begin{abstract}
Resumen
Las instituciones de educación superior - al igual que cualquier organización - necesitan de gestión estratégica de tecnologías de la información, la que permita alinear sus proyectos tecnológicos hacia el logro de los objetivos institucionales (Hinojo Lucena, Fernández Martín, \& Aznar Diaz, 2002). Muchas de estas ya poseen un plan estratégico institucional (PEI), pero este aún no termina de vincularse con los departamentos que lo conforman. La gestión de las tecnologías de la información y comunicación se maneja a través de un «Plan estratégico de tecnologías de información (PETI)» y surge de la necesidad de plantear proyectos tecnológicos que generen valor para una organización. Este artículo presenta la investigación realizada para el planteamiento de un modelo de PETI, para instituciones de educación superior, que se estableció con la ayuda de metodologías de gestión en tecnologías como: Auditoria en Gestión de las Tecnologías de la Información (MAIGTI), Information Technology Infraestructure Library (ITIL) (Orand, 2011), y Control Objectives for Information and related Technology (COBIT) (La De Haes, S; Van Grembergen, W; Debreceny, R.S, 2013), permitiendo así, analizar la organización en su conjunto a través del PEI, y el área de tecnologías de la información para plantear un PETI.

Palabras clave: Gestión estratégica de tecnologías de la información, PETI, PEI, MAIGTI, COBIT, ITIL, Educación superior, instituciones educativas, planeamiento.
\end{abstract}

\begin{abstract}
Institutions of higher education — as well as any other organization-, needs an 'strategic management of information technologies', which allow to align their technological projects for the achievement of institutional objectives. (Hinojo Lucena, Fernández Martín, \& Aznar Diaz, 2002). Many of these organizations already have an institutional strategic plan, but this has not yet been linked to their departments. The management of information and communication technologies is managed through an "Information Technology Strategic Plan (ITSP)" and arises from the need to propose technological projects that generate value for an organization. This article comes from a research where an ITSP model for higher education institutions was developed, which was established with the help of management methodologies in technologies such as: MAIGTI (Audit in Management of Information Technology), ITIL (Information Library of Technology Infrastructure) and COBIT (Control Objectives for Information and Related Technology) allowing thus analyze the organization as a whole through the Institutional Strategic Plan and then the area of information technologies through an ITSP.

Keywords: Information technology strategic plan, ITSP, ITIL, COBIT, MAIGTI, higher education institutions, planning.
\end{abstract}

Citar como: Choque, VMCh. (2018). Plan estratégico de tecnologías de la información orientado al éxito de proyectos en instituciones de educación superior. Rev Yachay, 7(1),424-434.

Recibido: 16-09-2018; Aceptado 07-12-2018

\section{Introducción}

El presente artículo se basa en el trabajo de investigación denominado «Modelo de plan estratégico de tecnologías de la información (TI) en instituciones de educación superior», trabajo en el cual se abordó la problemática de las áreas de tecnologías de la información al tener la necesidad de concebir proyectos y propuestas que generen valor para su organización y con ello logren el cumplimiento de los objetivos

Rev. Yachay volumen (7) Número (1), enero-diciembre 2018 
institucionales (Choque Soto, 2017). Se apostó por un plan estratégico, ya que actualmente la gran mayoría de instituciones poseen uno, pero el área de TI difícilmente está alineada a este y, por tanto, solo se limita a dar soporte a las actividades empresariales más, no a crear propuestas de valor. Este artículo plantea un plan estratégico de tecnologías de información basado en la información general de una universidad privada, la cual fue analizada a nivel organizacional y, a partir de este análisis, se plantearon propuestas de valor desde el área de TI, alineados a los objetivos instituciones (Wall, 1990).

\section{Materiales y métodos}

Para llevar a cabo las investigaciones y para el logro de los objetivos se comenzó por el análisis organizacional de una universidad local, para lo cual se utilizaron diferentes métodos que incluyeron técnicas como: la entrevista, la observación y la revisión documentaria; contando con materiales como la documentación oficial y de las áreas de tecnologías de la información, información de portales oficiales, páginas de transparencia, entre otros. En el desarrollo de la propuesta se utilizó la «Metodología de Auditoría en Gestión de las Tecnologías de la Información» MAIGTI (Alfaro Paredes, 2008), la cual a su vez, está basada en las metodologías internacionales Control Objectives for Information and related Technology COBIT de gobierno de TI, e Information Technology Infraestructure Library ITIL. MAIGTI se utilizó a lo largo de todo el proyecto, comenzando por el análisis previo al plan estratégico de tecnologías de la información, que incluyó el diagnóstico de la situación actual del área de TI, y con posterioridad proponer el modelo de plan estratégico de tecnologías de la información. En esta etapa se utilizó ITIL para la definición e implementación de estrategias de gestión de servicios de TI; para el diseño e implementación de los procesos y operación de servicios que el área brinda a la institución y, en la implementación y adaptación de políticas orientadas a la mejora continua de los servicios TI. De manera paralela se hizo uso de COBIT para analizar si los procesos, recursos informáticos y proyectos planteados realmente contribuyen al logro de los objetivos institucionales.

\section{Resultados}

Como resultado de la investigación se presentan los elementos más importantes del PETI formalmente propuesto:

\section{Estructura organizacional de la gestión de las tecnologías de información y comunicaciones}

Tomando como referencia el estándar COBIT y los procesos universitarios considerados importantes por las áreas de TI en general, se tiene el siguiente organigrama propuesto:

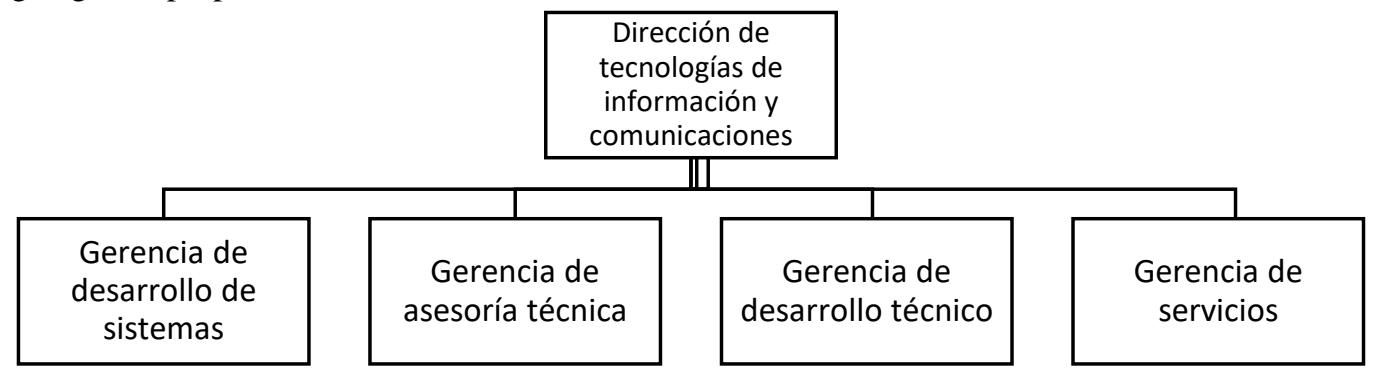

Ilustración 5: Organigrama Departamento de Tecnologías de la Información

Recursos humanos

- El director de tecnologías de la información, tiene una preparación integral en manejo y gestión de sistemas de información, administración de áreas de tecnologías de la información y centros de cómputo, gestión de redes computacionales, telecomunicaciones en general, internet y tecnologías de la información, de la misma manera, posee habilidades en administración general y de talento humano.

- Este director de sistemas de información deberá tener: capacidades gerenciales y técnicas. Su tarea es la de alinear el desarrollo de los sistemas de información con los planes de la compañía, así como también de elaborar y gestionar presupuestos y disponer de los equipos técnicos.

- El coordinador de sistemas: presta apoyo a la dirección de TI; formula propuestas, recomienda adquisiciones, evalúa técnicamente el software, coordina y supervisa la prestación de soporte, mantenimiento. (Muñoz Ortega, 2018) 
- Líder/guía de proyecto: encargado de identificar las necesidades de los usuarios y, posteriormente, gestionar los recursos humanos, económicos y materiales; de esta manera se busca obtener buenos resultados que cumplan la calidad necesaria y dentro del tiempo estimado.

- Desarrollador o programador: diseña y desarrolla aplicaciones; debe trasladar necesidades (requerimientos) en soluciones informáticas, escrita en un lenguaje informático.

- Asesor de métodos y procedimientos: encargado de hacer recomendaciones técnicas a los usuarios del sistema, de este modo, lograr sistematizar los requerimientos de información que mejoren los sistemas resultantes. Lo anterior se logra a través de la optimización de procedimientos reales de trabajo que incorporen sistemas y equipos de cómputo que los perfeccionen.

- Consultor técnico: responsable del funcionamiento efectivo del hardware y del software para los usuarios al administrar sistemas automatizados, independientes del computador central/servidor.

- Supervisor de operaciones: responsable de las operaciones del centro de cómputo o área de TI, encargado de cubrir los requerimientos específicos de servicio del turno al cual está asignado.

- Especialista operador de red y teleprocesos: responsable de planificar y dirigir las labores realizadas que tengan relación con redes cableadas e inalámbricas, para incorporar tecnología de punta, así como de brindar servicios de mantenimiento y actualización de todos los equipos de telecomunicaciones.

- Auxiliar de instalaciones: responsable de efectuar los planes de instalación de equipos y también de cumplir con las medidas de seguridad encargadas por el ingeniero a cargo del área de instalaciones.

\section{Infraestructura de tecnologías de información y comunicaciones (TIC)}

\section{Servidores}

Se contará con un total de 12 servidores.

\section{Almacenamiento}

- Almacenamiento SAN - Storage Area Network: con un total de 20 TB.

- Almacenamiento NAS - Network-Attached Storage: con un total 10 TB.

\section{Gestión centralizada de back ups}

- TSM - Tivoli Storage Manager: Capacidad de 25 TB.

- $\quad \mathrm{RBO}$ - Remote Backup Outsourcing: Capacidad de 75 GB.

\section{Racks (Soportes)}

Instalación de 14 racks.

\section{Puntos de red}

Capacidad total: 250 puntos de red.

\section{Operaciones de tecnologías de información y comunicaciones}

Operaciones a ser ejecutadas:

- Establecer un marco de gestión basado en mejores prácticas con métodos, técnicas, métricas y herramientas que permitan la eficiencia, eficacia y la optimización económica de los servicios de las tecnologías de la información y comunicaciones.

- Acrecentar la conectividad interna y externa de manera óptima y eficaz, segura y con buena disponibilidad. Se gestionará la adquisición de tecnologías de comunicación inalámbrica y móviles de alto rendimiento; también se pensará en implementar telefonía basada en servicios de internet y redes multiservicio (redes de datos, voz, video).

- Robustecer la infraestructura tecnológica para mejorar aspectos — como la eficiencia, disponibilidad, actualización y capacidad-. Con este fin, se incluirán los equipos principales, el software de soporte, el software de seguridad, altas capacidades de almacenamiento masivo, apoyo al usuario final y capacitación del recurso humano. 
- Implementar sistemas de información a través de la unificación de los macro procesos institucionales previamente analizados bajo estándares y herramientas.

- Los servicios de tercerización pueden ser una opción a considerar para el desarrollo de soluciones, dependiendo del proyecto y experiencia del personal en el área.

- Se implementará el servicio de 'mesa de ayuda' conforme al estándar ITIL, y se asimilará este modelo para mejorar la continuidad del negocio.

\section{Proyectos de tecnologías de información y comunicaciones}

- Implementación de sistema de biblioteca digital.

- Implementación de plataforma E-Learning Moodle.

- Implementación del ERP University.

- Implementación de comunicación por video/voz IP.

- Mejorar la infraestructura de redes.

- Renovación del parque de hardware.

- Implementación de un sistema de información para control y monitoreo de investigación universitaria.

Planes Operativos

\section{Planes de proyecto}

Se establecieron tres proyectos alineados a la estrategia general y que generaron valor institucional, de los cuales se desprende uno de los más representativos consecuencia de la evaluación del nivel de beneficio institucional. A continuación, se presenta la propuesta:

\section{Implementación del sistema de biblioteca digital}

\section{Objetivo}

El objetivo de la implementación del proyecto 'biblioteca digital' para las universidades es proveer los recursos tecnológicos para seleccionar, inspeccionar, salvaguardar la integridad y permitir un fácil acceso de la comunidad académica a colecciones de libros y demás material bibliográfico digital. Este proyecto se remonta, inclusive, a visiones de desarrollo tecnológico dese los años 90. (Muñoz, 1997)

\section{a) Alcance}

El proyecto considera la implementación de una biblioteca digital mediante el cual se podrá acceder a un acervo que contará con las colecciones disponibles de textos digitales, así como libros de diferentes materias de conocimiento.

\section{b) Justificación}

El proyecto resulta convergente con el objetivo estratégico de la empresa que es «Mejoramiento en innovación de servicios», y justifica su desarrollo ya que se destaca como herramienta para innovar la educación integral. (Estay, C, \& Pastor, J., 2002). Además, luego de la evaluación del beneficio de la implementación de la biblioteca digital, en el caso optimista significa un incremento de ingresos y clientes para la institución. 
Tabla 1: Evaluación del beneficio de implementación de la biblioteca digital

EVALUACIÓN DEL BENEFICIO DE LA IMPLEMENTACION DE BIBLIOTECA DIGITAL

CASO OPTIMISTA (S/-)

\begin{tabular}{|c|c|c|c|c|c|}
\hline & Año 0 & Año 1 & Año 2 & Año 3 & Año 4 \\
\hline \multicolumn{6}{|l|}{ Ingresos Adicionales } \\
\hline Margen de Contribución & & 96000 & 96000 & 96000 & 96000 \\
\hline Ahorros & & 50000 & 50000 & 50000 & 50000 \\
\hline Total & & 146000 & 146000 & 146000 & 146000 \\
\hline \multicolumn{6}{|l|}{ Egresos Adicionales } \\
\hline Inversiones & 80000 & & & & \\
\hline Gastos & 10000 & 30000 & 30000 & 30000 & 30000 \\
\hline Total & 90000 & 30000 & 30000 & 30000 & 30000 \\
\hline Flujo Neto & -90000 & 116000 & 116000 & 116000 & 116000 \\
\hline TMAR & $10 \%$ & & & & \\
\hline VNA 2 AÑOS & 111322 & & & & \\
\hline VNA 4 AÑOS & 277704 & & & & \\
\hline
\end{tabular}

c) Especificación de requerimientos funcionales y técnicos

- Requerimientos funcionales

Los requerimientos funcionales del proyecto son los siguientes:

$\checkmark$ El sistema deberá contar con un catálogo de libros que mostrará resultados de la búsqueda de estos por categoría y desplegará los detalles de cada texto seleccionado.

$\checkmark$ También deberá mostrar un cuadro de búsqueda con filtro de bibliotecas.

$\checkmark$ Además del catálogo, el usuario debe poder ver los libros ya descargados.

$\checkmark \quad$ Debe proveer también un sistema de seguridad que garantice los contenidos sujetos a derechos de autor.

- Requerimientos Técnicos

$\checkmark$ El sistema deberá ser accesible vía web y desarrollado con herramientas libres.

$\checkmark$ Deberá poder ser accesible en distintos sistemas operativos y plataformas de hardware. Los requisitos técnicos mínimos para Windows son:

- Los libros se podrán descargar en formato PDF y ser abiertos con Adobe Reader X o superior.

- Se podrá acceder al sistema mediante distintos navegadores, como Chrome, Internet Explorer 9.0 o superior, Firefox, Opera.

d) Organización del proyecto:

a. Equipo de informática:

El equipo de trabajo incluirá los siguientes responsables:

$\checkmark$ Coordinador técnico y documentalista e informática.

$\checkmark$ Responsable de selección de contenido y digitalización del acervo documentario.

$\checkmark$ Responsable de la maquetación e implementación web.

$\checkmark$ Responsable del desarrollo del proyecto.

b. Equipo de usuarios:

Todos los estudiantes, docentes, personal administrativo e integrantes de la institución.

c. Equipo auditor o supervisor:

Se contará con un responsable para realizar constante seguimiento para el cumplimiento de los requisitos y del desarrollo de software con calidad. 
e) Recursos necesarios:

Los recursos a utilizar incluyen 5 personas encargadas, un ambiente para el desarrollo de las actividades del proyecto y los recursos financieros incluirán, costo de implantación, mantenimiento anual, software de base.

f) Cronograma de ejecución considerando cadena crítica

\begin{tabular}{|c|c|c|c|c|c|c|c|c|c|c|c|c|c|}
\hline \multirow[t]{2}{*}{ Id } & \multirow[t]{2}{*}{ Nombre de tarea } & \multirow[t]{2}{*}{ Duración } & \multirow[t]{2}{*}{ Comienzo } & \multirow[t]{2}{*}{ Fin } & \multicolumn{4}{|c|}{$29 \operatorname{sep} ' 13$} & \multicolumn{4}{|c|}{13 oct ' 13} & \multirow{2}{*}{$\begin{array}{c}27 \text { oct } \\
D\end{array}$} \\
\hline & & & & & $\mathrm{x}$ & D & J & $\mathrm{L}$ & V & M & $\mathrm{S}$ & $\mathrm{x}$ & \\
\hline 1 & Requerimientos de los Usuarios & 5.5 días & $\operatorname{mar} 01 / 10 / 13$ & $\operatorname{mar} 08 / 10 / 13$ & & & & & & & & & \\
\hline 4 & Analisis de requisitos de software & 17 días & $\operatorname{mar} 08 / 10 / 13$ & jue $31 / 10 / 13$ & & & & & & & & & \\
\hline 11 & Analisis de requisitos del Sistema & 6 días & jue $31 / 10 / 13$ & vie $08 / 11 / 13$ & & & & & & & & & \\
\hline 14 & Diseño Preliminar & 13 días & vie $08 / 11 / 13$ & mié $27 / 11 / 13$ & & & & & & & & & \\
\hline 17 & Diseño Detallado & 5 días & mié 27/11/13 & mié 04/12/13 & & & & & & & & & \\
\hline 20 & Diseño de la Bases de Datos & 6 días & mié 04/12/13 & jue $12 / 12 / 13$ & & & & & & & & & \\
\hline 23 & ENTREGA DISEÑO & 0 días & jue $19 / 12 / 13$ & jue $19 / 12 / 13$ & & & & & & & & & \\
\hline 24 & Construccion & 19 días & jue $12 / 12 / 13$ & mié 08/01/14 & & & & & & & & & \\
\hline 28 & Pruebas & 12 dias & mié 08/01/14 & vie $24 / 01 / 14$ & & & & & & & & & \\
\hline 32 & Demostracion a los usuarios & 5 días & vie $24 / 01 / 14$ & vie $31 / 01 / 14$ & & & & & & & & & \\
\hline 35 & Reunion & 90.25 dias & $\operatorname{mar} 01 / 10 / 13$ & $\operatorname{mar} 04 / 02 / 14$ & & b & & & & b & & & 0 \\
\hline
\end{tabular}

Ilustración 1: Cronograma del proyecto

Plan de seguridad de la información

La comisión de seguridad de la información está conformada de la siguiente manera:

Tabla 2: Comisión de seguridad de la información

\begin{tabular}{ll}
\hline \multicolumn{1}{c}{ Área } & \multicolumn{1}{c}{ Representante } \\
\hline Secretaría de planificación y gestión institucional & Secretario \\
Coordinación de informática & Coordinador \\
Unidad de auditoría interna & Auditor interno titular \\
Seguridad física & Un representante designado por la comisión para la seguridad. \\
Seguridad de la información & Responsable de la seguridad de la información \\
\hline
\end{tabular}

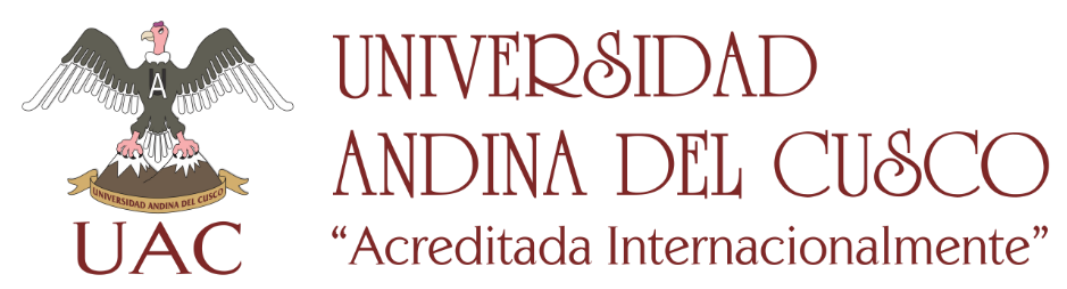

Rev. Yachay volumen (7) Número (1), enero-diciembre 2018 
Evaluación de riesgos de seguridad de la información

Tabla 3: Evaluación de Riesgos

\begin{tabular}{|c|c|c|c|}
\hline RIESGO/VULNERABILIDAD & Probabilidad & Impacto & $\begin{array}{c}\text { Valor } \\
\text { Esperado }\end{array}$ \\
\hline Interés en obtener información estratégica, por parte de comP.E.T.I.dores de negocio. & 2 & 4 & 8 \\
\hline Interés en obtener beneficios económicos mediante actividades dolosas. & 2 & 3 & 6 \\
\hline Actividad vandálica realizada por hackers o crackers & 3 & 4 & 12 \\
\hline Pérdida de información fruto de contaminación por virus informático. & 2 & 3 & 6 \\
\hline $\begin{array}{l}\text { Pérdida de información a través del personal que ingresa de manera transitoria en sustitución } \\
\text { de empleados en vacaciones. }\end{array}$ & 2 & 4 & 8 \\
\hline No se cuenta con un catálogo de perfiles de acceso a los sistemas. & 1 & 3 & 3 \\
\hline Desproporción de contraseñas manejadas por los usuarios. & 2 & 2 & 4 \\
\hline Usuarios temporales del área de desarrollo y personal con acceso al entorno de producción. & 2 & 3 & 6 \\
\hline Aplicaciones cuyo ingreso no es vigilado por el área de seguridad informática. & 2 & 3 & 6 \\
\hline Falta de conciencia en seguridad por parte del personal. & 2 & 4 & 8 \\
\hline Falta de revisión de la información que envían los usuarios hacia internet. & 2 & 4 & 8 \\
\hline No existen controles correctos para la información almacenada en los ordenadores. & 2 & 4 & 8 \\
\hline Arquitectura de red inadecuada para examinar accesos no permitidos desde redes externas. & 3 & 4 & 12 \\
\hline Fuga de información estratégica mediante remoción de computadores portátiles. & 1 & 4 & 4 \\
\hline Acceso no autorizado a través de redes inalámbricas sin protección. & 2 & 4 & 8 \\
\hline Inspecciones de acceso a internet desde la red privada. & 3 & 3 & 9 \\
\hline $\begin{array}{l}\text { Valores: } \\
1 \text { = Insignificante } \\
2 \text { = Baja } \\
3 \text { = Media } \\
4=\text { Alta } \\
\text { Alto riesgo (1 216), medio riesgo (89) y bajo riesgo (16) }\end{array}$ & & & \\
\hline
\end{tabular}

Rev. Yachay volumen (7) Número (1), enero-diciembre 2018 
Evaluación de la estrategia

Tabla 4: Evaluación de la estrategia

\begin{tabular}{|c|c|}
\hline INDICADOR & DESCRIPCIÓN \\
\hline Número de matriculados & $\begin{array}{l}\text { Procedimiento de cálculo: Medición directa } \\
\text { Unidad: Número } \\
\text { Sentido: Creciente } \\
\text { Fuente de información: Oficina de Admisión } \\
\text { Frecuencia: Semestral }\end{array}$ \\
\hline Número de ingresantes & $\begin{array}{l}\text { Procedimiento de cálculo: Medición directa } \\
\text { Unidad: Número } \\
\text { Sentido: Creciente } \\
\text { Fuente de información: Oficina de Admisión } \\
\text { Frecuencia: Semestral }\end{array}$ \\
\hline Número de graduados & $\begin{array}{l}\text { Procedimiento de cálculo: Medición directa } \\
\text { Unidad: Número } \\
\text { Sentido: Creciente } \\
\text { Fuente de información: Oficina de Grados y Títulos } \\
\text { Frecuencia: Semestral }\end{array}$ \\
\hline Porcentaje de graduados & $\begin{array}{l}\text { Procedimiento de cálculo: Fórmula } \\
=\left(\mathrm{N}^{\circ} \text { de Graduados } \mathrm{N} .^{\circ} \text { de ingresantes }\right)^{*} 100 \% \\
\text { Unidad: Porcentaje } \\
\text { Sentido: Creciente } \\
\text { Fuente de información: Oficina de Admisión, Grados y Títulos } \\
\text { Frecuencia: Semestral }\end{array}$ \\
\hline Número de alumnos de posgrado & $\begin{array}{l}\text { Procedimiento de cálculo: Medición directa } \\
\text { Unidad: Número } \\
\text { Sentido: Creciente } \\
\text { Fuente de información: Unidades de posgrado } \\
\text { Frecuencia: Semestral }\end{array}$ \\
\hline Margen de Utilidad & $\begin{array}{l}\text { Procedimiento de cálculo: Fórmula } \\
\qquad=\text { (ingresos }- \text { egresos) } \\
\text { Unidad: Moneda } \\
\text { Sentido: Creciente } \\
\text { Fuente de información: Oficina financiera } \\
\text { Frecuencia: Anual }\end{array}$ \\
\hline
\end{tabular}




\begin{tabular}{|c|c|}
\hline Índice de ejecución presupuestaria & $\begin{array}{l}\text { Procedimiento de cálculo: Fórmula } \\
\qquad \quad=(\text { Pres. Ejecutado/ Pres. Asignado })^{*} 100 \% \\
\text { Unidad: Porcentaje } \\
\text { Sentido: Creciente } \\
\text { Fuente de información: Oficina Financiera } \\
\text { Frecuencia: Anual }\end{array}$ \\
\hline Número de carreras nuevas & $\begin{array}{l}\text { Procedimiento de cálculo: Medición directa } \\
\text { Unidad: Número } \\
\text { Sentido: Creciente } \\
\text { Fuente de información: Asamblea universitaria } \\
\text { Frecuencia: Anual }\end{array}$ \\
\hline
\end{tabular}

Tabla 5: Evaluación y comparación de la estrategia

\begin{tabular}{|c|c|c|c|c|c|c|c|c|c|c|}
\hline ESTRATEGI & INICIATIV & INDICADOR & UNIDA & TIPO DE & FRECUENCI & & & $\overline{\text { META }}$ & & \\
\hline A & $\mathbf{A}$ & & D & INDICADO & A & AÑO & AÑO & AÑ̃O & AÑO & AÑO \\
\hline & & & & $\mathbf{R}$ & & 01 & 02 & $\mathbf{0 3}$ & 04 & 05 \\
\hline \multirow{8}{*}{$\begin{array}{l}\text { Mejoramiento } \\
\text { e innovación } \\
\text { de servicios }\end{array}$} & \multirow[t]{6}{*}{$\begin{array}{l}\text { Ampliación } \\
\text { de Mercado }\end{array}$} & $\begin{array}{l}\text { Número de } \\
\text { matriculados }\end{array}$ & $\mathrm{N} \cdot{ }^{\circ}$ & Simple & Semestral & $2 \%$ & $5 \%$ & $5 \%$ & $7 \%$ & $10 \%$ \\
\hline & & Número de ingresantes & $\mathrm{N} \cdot{ }^{\circ}$ & Simple & Semestral & $2 \%$ & $3 \%$ & $5 \%$ & $7 \%$ & $10 \%$ \\
\hline & & Número de graduados & $\mathrm{N} \cdot{ }^{\circ}$ & Simple & Semestral & $2 \%$ & $2 \%$ & $3 \%$ & $3 \%$ & $5 \%$ \\
\hline & & $\begin{array}{l}\text { Porcentaje de } \\
\text { graduados }\end{array}$ & $\%$ & Compuesto & Semestral & $2 \%$ & $2 \%$ & $3 \%$ & $4 \%$ & $5 \%$ \\
\hline & & $\begin{array}{l}\text { Número de alumnos de } \\
\text { posgrado }\end{array}$ & $\mathrm{N} .{ }^{\circ}$ & Simple & Semestral & $2 \%$ & $2 \%$ & $3 \%$ & $4 \%$ & $5 \%$ \\
\hline & & $\begin{array}{l}\text { Número de carreras } \\
\text { nuevas }\end{array}$ & $\mathrm{N} .{ }^{\circ}$ & Simple & Anual & 0 & 0 & 0 & 1 & 1 \\
\hline & \multirow{2}{*}{$\begin{array}{c}\text { Incrementar } \\
\text { ingresos }\end{array}$} & Margen de utilidad & Moneda & Compuesto & Anual & $5 \%$ & $5 \%$ & $7 \%$ & $10 \%$ & $12 \%$ \\
\hline & & $\begin{array}{l}\text { Índice de ejecución } \\
\text { Presupuestaria }\end{array}$ & $\%$ & Compuesto & Anual & $10 \%$ & $12 \%$ & $15 \%$ & $17 \%$ & $20 \%$ \\
\hline
\end{tabular}

\section{Discusión}

El resultado final de esta investigación fue, principalmente, toda la documentación formal presentada como modelo del 'plan estratégico de tecnologías de la información' (PETI), del cual se presenta solo un extracto en el presente artículo. Como podemos ver se presentan 4 
puntos muy importantes: la estructura organizacional del departamento de TI, los recursos humanos necesarios, la infraestructura necesaria y los proyectos propuestos y, el plan de seguridad de la información; todos ellos elaborados demostrando la aplicación de las distintas metodologías de gestión estratégica de TI antes mencionadas. En el trabajo de investigación original, se presenta todo el alcance del PETI elaborado para mayor información (Choque Soto, 2017).

Las instituciones no son conscientes de la importancia de un PETI para el cumplimiento de sus objetivos, así como hace algunos años tampoco consideraban la importancia de un PEI, pero ahora se ha vuelto muy común en ellas y es establecida de manera seria y periódica, por tanto, es importante cambiar la forma de pensar de las instituciones, trabajar bajo un enfoque más horizontal por procesos, que es una de las propuestas de la presente investigación y, de esta manera, comenzar a pensar en alinear los proyectos de TI hacia los objetivos institucionales, de manera que realmente generen valor y beneficios para las instituciones.

\section{Conclusiones}

- Se puede concluir que lo propuesto en el PETI final como modelo es adaptable para cualquier institución educativa de nivel superior.

- El utilizar metodologías reconocidas a nivel internacional, permite estandarizar los elementos que un PETI debería contener.

- Permite otorgar los lineamientos y bases para realizar un posterior seguimiento y monitorización de su cumplimiento.

\section{Recomendaciones}

- Aún queda por aclarar el proceso de implementación de la estrategia y sus resultados pero, esa decisión escapa de las manos del investigador, dependerá de la decisión institucional de adoptarla y ejecutarla para los próximos cuatro años, lo cual sería fructífero para el crecimiento de la presente propuesta y el cumplimento de sus objetivos a largo plazo.

- Se sugiere - dentro del marco de la gestión estratégica de tecnologías de la información — plantear también un modelo de seguimiento o control para la mejora continua de planes estratégicos de TI. Se propone para esto, el uso de un BAM (Business Activity Monitoring) (Kolar, 2009), que esté adaptado a las actividades y los procesos de la institución, que permita su monitorización y seguimiento para el cumplimiento del PETI.

\section{Referencias Bibliográficas}

Alfaro Paredes, E. (2008). Metodología para la auditoría integral de la gestión de la tecnología de información. Pontificia Universidad Católica del Perú, Lima. Lima : PUCP. Recuperado el 3 de enero de 2019, de http://tesis.pucp.edu.pe/repositorio/bitstream/handle/123456789/1048/ALFARO_PAREDES_EMIGDIO_AUDITORIA_GESTI ON_TECNOLOGIA_INFORMACION.pdf?sequence=1\&isAllowed=y

Choque Soto, V. (2017). Modelo de Plan Estratégico de Tecnologías de la Información y Comunicacion. Tesis de grado, INSTITUTO CIENTÍFICO Y TECNOLÓGICO DEL EJÉRCITO, ESCUELA DE POSGRADO, Lima, Lima Peru. Recuperado el 3 de enero de 2019, de http://repositorio.icte.ejercito.mil.pe/bitstream/ICTE/36/1/Tesis\%20Bach\%20\%20Choque\%20Soto\%20Vanessa\%20Maribel.pd f

E. Mallo, P., Artola, M., Zanfrillo, A., Morettin, M., Galante, M., E. Pascual, M., \& Busetto, A. (2006). Aspirantes a una institución de educación superior. Economia UNMDP, 27-40.

Hinojo Lucena, F., Fernández Martín, F., \& Aznar Diaz, L. (2002). Las actitudes de los docentes hacia la formación en tecnologías de la información y comunicación (TIC) aplicadas a la educación. Revista de educación(5), 253-270. Recuperado el 2 de enero de 2019, de https://dialnet.unirioja.es/servlet/articulo?codigo=498346

Kolar, J. (2009). Business activity monitoring. República Chec: Masaryk University.

La De Haes, S; Van Grembergen, W; Debreceny, R.S. (2013). COBIT 5 and enterprise governance of information technology: Building blocks and research opportunities. Journal of Information Systems, 27(1), 307-324. doi:https://doi.org/10.2308/isys-50422

Muñoz Ortega, G. (14 de 09 de 2018). DNDA. Obtenido de http://derechodeautor.gov.co/perfil-coordinador-sistemas 
Muñoz, O. (1997). El desarrollo tecnológico como objetivo estratégico. (O. Muñoz, Ed.) Santiago de Compostela, España: Ed.

Universidad de Santiago \& Instituto de estudios avanzados. Recuperado el 3 de enero de 2019

NDP. (8 de junio de 2017). Agencia Peruana de Noticias; . Obtenido de http://andina.pe/agencia/noticia.aspx?id=670189

Orand, B. (2011). Foundations of IT service management: With ITIL 2011 (2.da ed.). (J. Villarreal, Ed.) USA: ITILyaBrady. Recuperado el 2 de enero de 2019, de https://www.amazon.com/Foundations-Service-Management-ITIL-2011/dp/1466231327

Wall, J. F. (1990). Alfred I. du Pont: The Man and His Family. Wilmington, USA: NN. Obtenido de https://www.amazon.com/Alfred-IPont-Man-Family/dp/0195043499
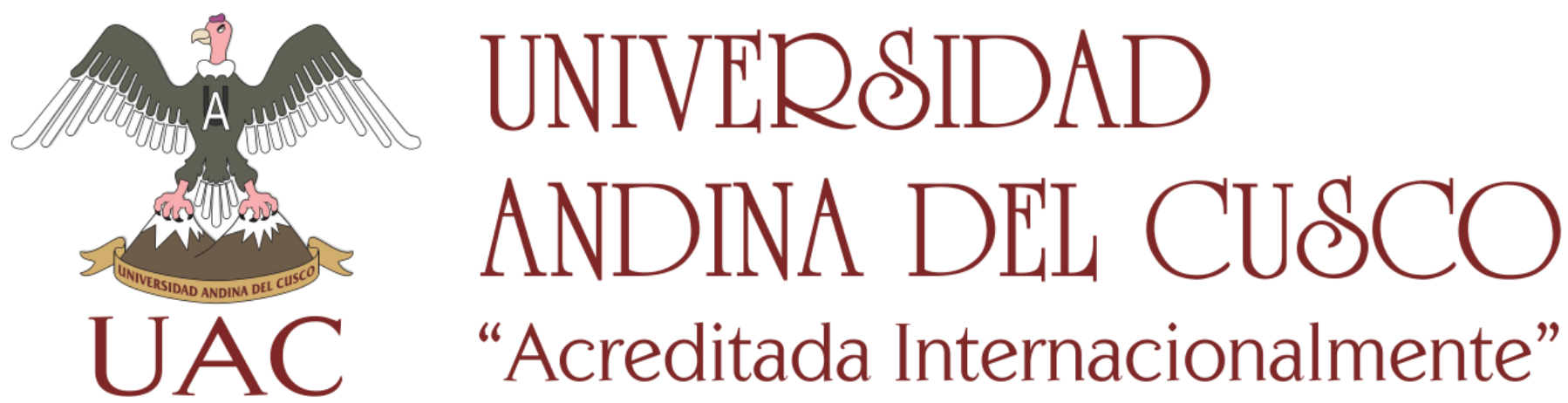

"Acreditada Internacionalmente" 